\title{
A Fast Matching Algorithm with Feature Points Based on NCC
}

\author{
Bo Yi, Ping Yu*, Guo-zhu He*, Jing Chen \\ Sichuan Agricultural University \\ Dujiangyan, China \\ heguozhu@sicau.edu,cn
}

\begin{abstract}
Featured with high anti-noise ability and precision, normalized cross-correlation (NCC) algorithm is broadly used in the feature point matching. However, it's not applied to the real-time processing because of the complexity of searching procedure and the great calculation quantity. To solve these problems, an improved NCC matching procedure is provided in this paper. First, the direction parameters of the feature points are abstracted with the histogram of gradient orientation (HOG), the pairs of the feature point in different gradient direction are filtered, and the matching matrix is used to get the right matching pair in order to avoid the repeated searching procedure. It can be included from the experiment result that the algorithm is not only characterized with good matching efficiency, but also can improve the calculation speed, which is good for the real-time processing and image matching with small amount of rotation.
\end{abstract}

Keywords-Image matching; feature point; normalized cross-correlation(NCC); histogram of gradient orientation (HOG); matching matrix

\section{INTRODUCTION}

As the key for the image splice technology, the image matching is used to find one spatial transformation relation for the matching of the coordinate points one by one in the overlapped images. The technology is widely used in the computer vision, remote sensing image analysis, pattern recognition, medical image processing and military field. There are two main methods for the image matching: area-based matching [1] and feature-based matching [2]. And the feature-based matching technology is now used mainly by the researchers in the world. The image features include feature points, line segments, margins, outlines, closed areas, etc. This method is applied to the complex matching and the real-time processing because of its small amount of calculation, good ability of robustness and low influence by the image light and brightness

The feature point matching based on the normalized cross-correlation (NCC) is well employed because of its good anti-noise ability and thus is of importance in the image matching. However, large amount of calculations are necessary for this method, and longer calculation time is needed by more feature points. Many researches mainly focusing on the template-based matching have been carried out to solve the problem of low efficiency of the NCC algorithm. The templates with the highest similarity within the images to be matched are figured out according to the known image templates in the research. Such methods as simplification and acceleration of the NCC matching with the convolution principle by Sun Bojiao [3]; reduction of the calculation amount through decreasing the search space with the combination of characteristics of area centroid, color histogram and NCC algorithm by Hu Min[4]; reduction of calculation amount with polynomial approximation template images by Shinichiro OMACHF [5] are not applied to NCC matching based on feature point.

In the matching of feature points with the traditional NCC method, it is necessary to calculate the correlation coefficient of every pair of the feature points in the point set for many times. The points with correlation coefficients less than the threshold values in the point set are filtered successively to determine the final matching pairs. The matching result and speed of the calculation method are affected by the threshold value and the number of the feature points.

It is proposed in this paper the feature points be abstracted with the Harris operator, the number of the feature points be controlled with Harris' corner response function, the main direction of every feature point be determined and compared according to the principles of the Histogram of Oriented Gradient (HOG), then the pairs of feature points whose similarity coefficients are not necessary to be calculated be filtered. Many times of repeated searching procedure can thus be avoided by building the matching matrix when the correlation coefficients of every pair of the feature points in the set are calculated. It has been shown in the test that the calculation time can be greatly shortened with this method while the matching precise can be ensured.

\section{EXTRACTION OF HarRis FEATURE PoINTS}

Harris Operator [6] is characterized with good rotatability and affine invariance, and robust to noise, changes of brightness and vision of the image. This method is proposed on the basis of Moravec Algorithm. The matrix $M$ can express analytically the edge feature and the characteristics of the feature points by differential operation and the corner detection with autocorrelation matrix $M$.

$$
\begin{aligned}
& M=\sum_{u, v} w(u, v)\left[\begin{array}{cc}
f_{x}^{2} & f_{x} f_{y} \\
f_{x} f_{y} & f_{y}^{2}
\end{array}\right] \\
& w(u, v)=\exp \left(-\frac{u^{2}+v^{2}}{2 \sigma^{2}}\right)
\end{aligned}
$$

Thereinto, $w(u, v)$ is the coefficient of the Gauss window at $(u, v)$ with the performance of image smoothness 
and noise reduction; $f_{x}$ and $f_{y}$ are the horizontal and vertical gradients respectively. When the feature values $l_{1}$ and $l_{2}$ are comparatively great, the feature points are determined. In order to avoid the direct solution of the matrix's feature value, Harris made the following corner response function:

$$
R=\operatorname{det}(M)-k \operatorname{trace}^{2}(M)
$$

Thereinto, det is the determinant of the autocorrelation matrix $M$, trace is the trajectory of $M$ (sum of diagonal elements of the matrix), and $K$ is a constant that is suggested to be between $0.04 \sim 0.06$. When the value of $R$ is greater than the threshold $T$ and reaches the local maximum, this point is taken as the feature point. In order to test the impact of the feature point number on the NCC matching efficiency, the response values $R$ of all the feature points are sorted in the descending order [7], and the point with the great response value is taken as the feature point that needs be matched.

\section{NCC MATCHING}

\section{A. Traditional NCC Matching Procedure}

NCC matching is well developed and often used now for the feature point matching in most of the image stitching algorithm [8]. NCC matching is characteristic with easy operation and good anti-noise ability. The relevant function is defined as:

$$
N O C=\frac{\sum_{i=1}^{N} \sum_{j=1}^{N}((x-i, y-\jmath)-\bar{I})\left(I\left(x^{\prime}-i, y^{\prime}-\jmath\right)-\bar{I}\right)}{\sqrt{\left.\sum_{i=1}^{N} \sum_{j=1}^{N}(I x-i, y-\jmath)-I^{2}\right)^{N} \sum_{i=1}^{N}\left(I\left(x^{\prime}-i, y^{\prime}-\jmath\right)-\bar{I}\right)^{2}}}
$$

Thereinto, the neighborhood size of the feature point is $N \times N, I$ and $I \notin$ represent the gray-scale values of the two images respectively, and $\bar{I}$ and $\bar{I} \mathscr{\phi}$ are the average gray-scale values of all the pixels within the feature point field of the two images respectively. The greater the NCC value is, the better similarity of the images will be.

The calculation procedure is as follows.

1) Every one of the feature points in image $I$ can be taken as the center for the choice of the neighborhood window of $N \times N$. The correlation calculations between this window and the neighborhood windows with the same size of all the feature points in image $I \Phi$ are carried out. And the feature point with the greatest correlation coefficient, and greater than the given threshold, is taken as the matching point. Thus, the first group of matching point set is determined.

2) The matching point for every one of the feature points in image $I \mathbb{\Phi}$ can be found according to the procedures in step 1 and the second matching point set is obtained.

3) Two groups of matching point sets can be determined after the bi-directional searching, from which all the same matching pairs can be found for the last matching results.

The above calculation procedure can be affected greatly by the threshold and the feature point number, so lots of intermediate results and invalid matching pairs in the matching procedure will be produced.

\section{B. Improved NCC Matching Procedure}

1) Direction parameter of feature points

The SIFT (scale invariant feature transform) algorithm[9] was improved in 2004 by D.G. Lowe. It is pointed out in this method that the direction parameter of every feature point can be determined with the characteristics of HOG and the operator is provided with rotational invariance. The direction parameters of the feature points are compared and the feature point pairs with different gradient directions are filtered to reduce the amount of calculation of NCC.

It is pointed out by Lowe that the image can be smoothed with the Gaussian function for the noise reduction before the calculations of modulus and direction of the feature point gradient.

$$
L(x, y)=G(x, y, s) \quad f(x, y)
$$

Thereinto, $G$ is for Gaussian function, $\sigma$ can determine the image smoothness and $\otimes$ is for the convolution operation.

For the image $L$ after the Gaussian smoothness, the gradient modulus and direction of the feature points can be calculated with Formula (6) and Formula (7).

$$
\begin{gathered}
m(x, y)=\sqrt{(L(x+1, y)-L(x-1, y))^{2}+(L(x, y+1)-L(x, y-1))^{2}} \\
\theta(x, y)=\arctan \frac{L(x, y+1)-L(x, y-1)}{L(x+1, y)-L(x-1, y)}
\end{gathered}
$$

For the determination of the main direction of every feature point, the gradient modulus and direction value will be firstly calculated within the neighborhood centering on the feature point. The gradient direction range is within $0 \sim 360^{\circ}$ expressed with histograms. There are totally $H$ columns, every of which represents the directional interval of $\theta$. The gradient direction of every pixel within this neighborhood is determined, and the gradient modulus of the pixels in the same direction is Gauss weighted and accumulated to the interval where they belong to. And the value of the main peak in the histogram is chosen as the main direction of the feature point.

\section{2) Improved matching procedure}

The matching can be filtered by comparing directly the direction parameters of the feature point, which is applied to such situation that there are no big rotation angles between the images. The total number of the feature points is set as $m$ in Image $I$, and $n$ in $I \mathbb{\complement}$. The matching matrix $H$ with the size of $m \times n$ is set.

The calculation procedures are as follows. 
a) The main direction of every feature point is firstly determined, where the HOG is set as $H=9, \theta=40^{\circ}$.

b) The main direction of any feature point $i(i=1,2, \ldots, m)$ in image $I$ to be matched is set as $\theta_{i}$. Every feature point $j(j=1,2, \cdots, n)$ in image $I \Phi$ is chosen successively, and the main direction is set as $\theta_{j}$. If $\theta_{i}=\theta_{j}$, the correlation coefficient of the feature point pair $C_{i j}$ is calculated and put into the matching matrix $H(i, j)$. If $\theta_{i} \neq \theta_{j}, \quad H(i, j)=0$.

c) Maximal value $C_{i}$ of the column in the matching matrix $H$ is searched. If $C_{i}$ is greater than the given threshold, the maximal value $C_{j}$ of the line where $C_{i}$ exists will be found. If $C_{i}=C_{j}$, the feature point $i$ in image $I$ and the feature point $j$ in image $I \Phi$ are taken as the matching points.

The initial matching sets can be obtained after the NCC matching. However, there are usually some false matching pairs of the feature points, which can be omitted with random sample consensus (RANSAC [10]) of good robustness, thus to get the final matching sets.

\section{Simulation Test}

The processor of $2.10 \mathrm{GHz}$ Inter, memory of 2GB, operating system of Windows 7 vista and the programming of MATLAB 2011b are necessary for the test. The efficiency and validity of the improved algorithm are proved in many simulation tests from which two groups of tests are chosen. The number produced by the feature points are controlled by the corner response function of Harris Operator. And the calculation efficiency of the two algorithms at different numbers of feature points can be compared and analyzed.

\section{A. Simulation Test 1}

The sizes of the two images are $427 \times 320$ and the numbers of the feature point are chosen as 200, 400, 600, 800 and 1000 separately. The matching results of the two algorithms at the feature point number of 400 are shown in Table 1.26 pairs of correct matching points are extracted with the traditional method and 23 pairs of correct matching points are extracted with the improved method. More correct matching points are obtained with larger number of feature points, but the operating efficiency of the procedure is also affected heavily. It is concluded that good matching results can be produced with the two methods.

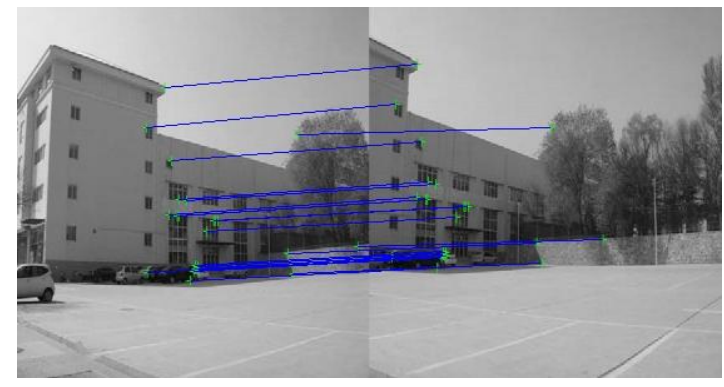

Figure .1 Matching result with traditional algorithm

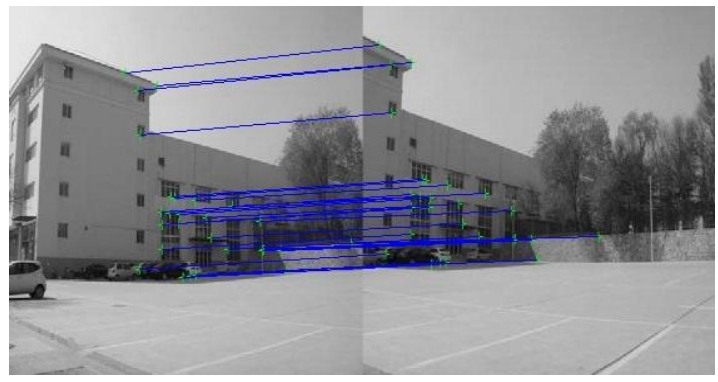

Figure .2 Matching result with improved algorithm

Table 1 Matching results of Test 1 at feature point of 400

For better precision of the test results, the grouped test is carried out for 10 times and the average value is calculated. The calculation time with the two algorithms are shown in Table 1 . The average time ratio between the improved algorithm and the traditional algorithm is 1:22. The greater number of the feature points is, the longer matching time with the traditional NCC will be obviously.

TABLE I. MAtChing Time IN TeSt 1

\begin{tabular}{cccccc}
\hline & \multicolumn{5}{c}{ Number of feature points } \\
\cline { 2 - 6 } & 200 & 400 & 600 & 800 & 1000 \\
\hline $\begin{array}{c}\text { Traditional } \\
\text { algorithm } \\
\text { (Times) }\end{array}$ & 0.9531 & 3.8205 & 8.5158 & 14.8886 & 22.5288 \\
$\begin{array}{c}\text { Improved } \\
\text { algorithm } \\
\text { (Time s) }\end{array}$ & 0.0442 & 0.1769 & 0.4006 & 0.6557 & 0.9459 \\
\hline Time ratio & 21.5633 & 21.5969 & 21.2576 & 22.7064 & 23.8173 \\
\hline
\end{tabular}

\section{B. Simulation Test 2}

The sizes of the two images are $256 \times 192$ and the detailed procedures are the same as those of Test 1.17 pairs of correct matching are obtained with the traditional method and 10 pairs of correct matching are obtained with the improve method according to the matching results from the two methods at the feature point number of 200. Please see Table 2.

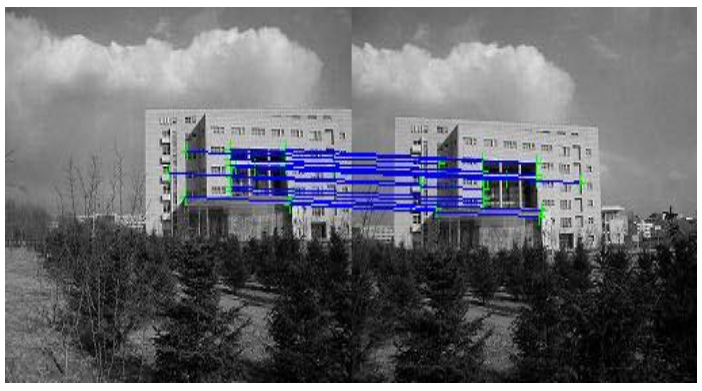

Figure .3 Matching result with traditional algorithm 


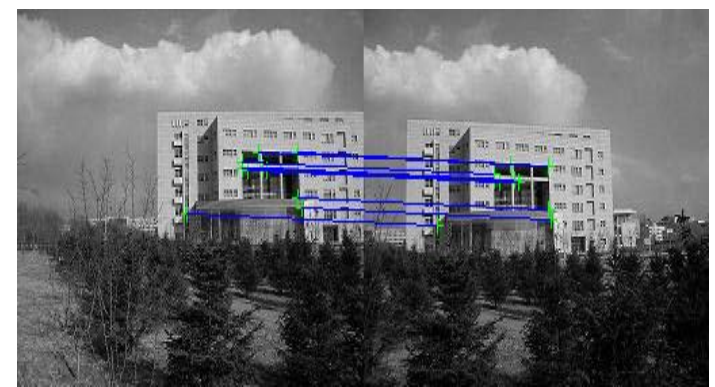

Figure $4 \quad$ Matching result with improved algorithm

Table 2 Matching results of Test 2 at feature point of 200

The matching time with the two algorithms at the different number of feature points are shown in Table 2. And the average time ratio of the two algorithms is $1: 28$. In the two group tests, the operation time with the traditional method at the same number of the feature points is more or less the same. However, the operating time with the improved algorithms is advanced obviously.

TABLE II. MATCHING TIME IN TEST 2

\begin{tabular}{cccccc}
\hline & \multicolumn{5}{c}{ Number of feature points } \\
\cline { 2 - 6 } & 200 & 400 & 600 & 800 & 1000 \\
\hline $\begin{array}{c}\text { Traditional } \\
\text { algorithm } \\
\text { (Times) }\end{array}$ & 0.9953 & 3.8690 & 8.5969 & 14.9898 & 23.6009 \\
$\begin{array}{c}\text { Improved } \\
\text { algorithm } \\
\text { (Time s) }\end{array}$ & 0.0707 & 0.1543 & 0.2859 & 0.4194 & 0.6081 \\
\hline Time ratio & 14.07316 & 25.07453 & 30.07381 & 35.7453 & 38.8134 \\
\hline
\end{tabular}

\section{CONCLUSION}

The matching procedure in this paper has been improved in view of much time for the feature point matching with the traditional NCC algorithm. The time lost in the bi-directional searching can be avoided efficiently by the decision of the main direction of the feature points with HOG, the filtration of the feature points in different directions and then the construction of the matching matrix. And the efficiency of the improved algorithm is proved from many simulation tests. However, the improved algorithm in this paper is only applied to the image matching with small rotation angle, because the NCC is not provided with rotational invariance. It will be considered in the future to make the NCC algorithm provided with rotational invariance by the application of the characteristics of the HOG and of the rotation compensation.

\section{ACKNOWLEDGEMENTS}

*Correspongding Author: $\mathrm{He}$ guo-zhu and $\mathrm{Yu}$ Ping .Sichuan Agricultural University, 611830.

\section{REFERENCES}

[1] B. S. Reddy, B. N. Chatterji. An FFT-Based Technique for Translation Rotation, and Scale-Invariant Image Registration. IEEE Transactions on Image Processing. 1996, 3(8): 1266-1270.

[2] David G. Lowe. Distinctive image features from scale-invariant key points. International Journal of Computer Vision, 2004, 60(2): 91-110.

[3] Dun Bojiao, Zhou Donghua. Fast matching method based on NCC Transducer and Microsystem Technologies. 2007, 26(9): 104-106.

[4] Hu Min, He Xiaojia, Wang Xiaohu. Fast Image Matching Algorithm with Area Centroid. Journal of Electronic Measurement and Instrument. 2011, 25(5): 455-462.

[5] Shinichiro OMACHF, Masako OMACHI. Fast template matching with polynomials [J]. IEEE Transactions On Image Processing, 2007, 16(8): 2139-2148.

[6] Harris C, Stephens M J. A Combined Corner and Edge Detector[C]. The 4th Alvey Vision Conf. Sheffield, UK: University of Sheffield Printing Unit, 1988: 147-151.

[7] Zhao Feng, Huang Qingming, Gao Wen. An Image Matching Algorithm Based on Singular Value Decomposition. Journal of Computer Research and Development. 2010, 47(1):23-32.

[8] Shi Xiangrong. Research on Video Stitching Based on Feature Points. North University of China, Taiyuan. 2012.

[9] David G. Lowe. Distinctive Image Features from Scale-Invariant key-points [J]. International Journal of Computer Vision, 2004, 60(2):91-110.

[10] Martin A. Fischler, Robert C. Bolles. Random Sample Consensus: A paradigm for model fitting with applications to image analysis and automated cartography [J]. Communications of the ACM, 1981, 24(6):381-395. 